\title{
A Compact Single-Feed Circularly Polarized Microstrip Antenna with Symmetric and Wide-Beamwidth Radiation Pattern
}

\author{
Xihong Ye, Mang He, Pingyuan Zhou, and Houjun Sun \\ School of Information and Electronics, Beijing Institute of Technology, Beijing 100081, China \\ Correspondence should be addressed to Mang He; hemang@bit.edu.cn
}

Received 18 July 2013; Revised 9 October 2013; Accepted 9 October 2013

Academic Editor: Michael Yan Wah Chia

Copyright (C) 2013 Xihong Ye et al. This is an open access article distributed under the Creative Commons Attribution License, which permits unrestricted use, distribution, and reproduction in any medium, provided the original work is properly cited.

\begin{abstract}
A compact single-feed circularly polarized microstrip antenna is proposed to achieve symmetric radiation pattern over a wide range of observation angles. In order to reduce the radiation aperture and consequently broaden the circular polarization (CP) and the half power beamwidth (HPBW) of the antenna, a partially etched superstrate and a conducting cavity are employed in the design. Further, reasonable axial ratio (AR) and impedance bandwidths are realized within the compact structure by using a simple series crossed-slot aperture coupled feeding. As a consequence, the overall dimension of the fabricated prototype is $0.32 \lambda_{0} \times 0.32 \lambda_{0} \times 0.12 \lambda_{0}$ at the center operating frequency of $1.56 \mathrm{GHz}$, and a $3.0 \%$ overlapped bandwidth of $10 \mathrm{~dB}$ return loss $(\mathrm{RL})$ and $3 \mathrm{~dB} A R$ is obtained. Within the bandwidth, symmetric $\mathrm{CP}$ radiation pattern over almost the entire upper hemisphere is observed and the HPBW is also increased from $60^{\circ}$ to $106^{\circ}$.
\end{abstract}

\section{Introduction}

Microstrip antennas (MSA) are widely used due to the advantages such as low profile, light weight, and easy conformability to host vehicles [1]. In many applications, the requirement of circularly polarized MSA with a wide field of views becomes more demanding in mobile base stations, wide-angle coverage satellite communications, high-resolution radar systems, and so on. For example, a GPS antenna for marine navigation should have good CP performance, adequate gain at low elevation angle, and symmetric and stable radiation at any elevation plane from about $10^{\circ}$ above the horizon to the zenith in order to stabilize the receiving signals. As well, compactsize design is often desired in such applications. In general, circular polarization designs can be achieved by using either single or multiple feeding structures [1-9]. For CP antennas of the former type, $3 \mathrm{~dB}$ AR beamwidth can be over $60^{\circ}$, but typically the usable overlapped impedance and AR bandwidths are less than $1 \%[1,4]$. Moreover, due to the inherent asymmetry in the single-feeding structure, symmetric radiation pattern is usually difficult to produce, while, in the latter category, $\mathrm{CP}$ radiation is generated by using a relatively complicated feeding network, such as the hybrid or power divider, to enhance the AR bandwidth but at the expense of increased manufacturing cost.

To realize symmetric and wide-beamwidth radiation, extensive researches have been conducted. Duan et al. designed a circular MSA with wide beamwidth by cutting out two symmetrical arc slots and loading three stubs on the circular patch [10]. The method extends the HPBW to $120^{\circ}$ effectively, but the antenna is linearly polarized. In $[2,3]$, a three-layer ground plane (GP) with gradually increased sizes from top to bottom and a pyramid-shape GP were used to broaden the CP beamwidth of MSAs, respectively. However, the use of three-dimensional GP structures increases the profile and lateral sizes of the MSA, which may limit their applications in practicing engineering. In [5-7], the AR bandwidths of MSAs had been extended by using multiple feed techniques, but the HPBW was narrow with the gain at small elevation angle being quite low, which cannot meet the requirements in navigation systems. Moreover, symmetrical radiations on different elevation cuts were not maintained, which may also be a pitfall to avoid in the practical designs. In [8], Nakano et al. utilized a folded conducting wall to partially enclose a probe-fed CP MSA with truncated corners in order to broaden the HPBW to $105^{\circ}$ that is approximately $40^{\circ}$ wider 


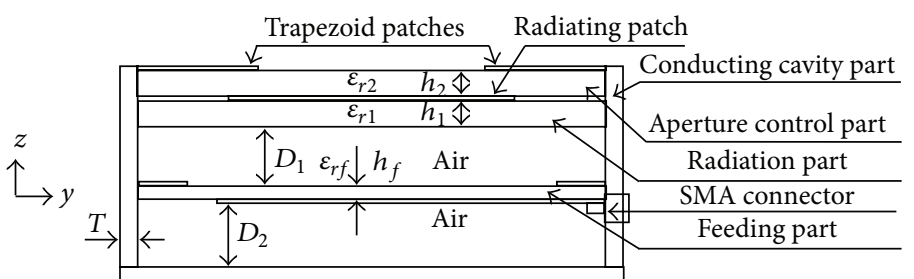

(a) Sectional view

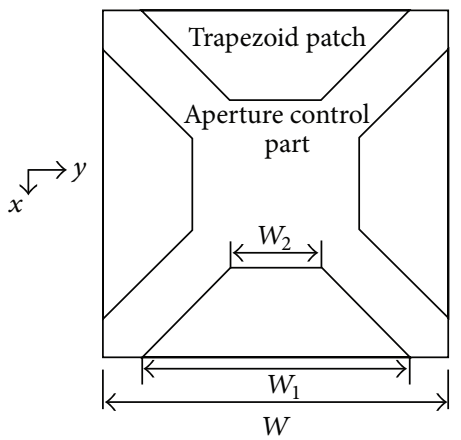

(c) Aperture control part

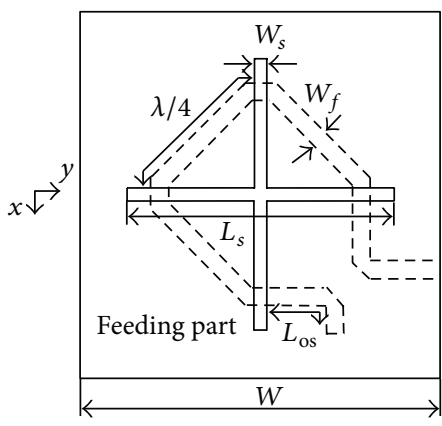

(d) Feeding part

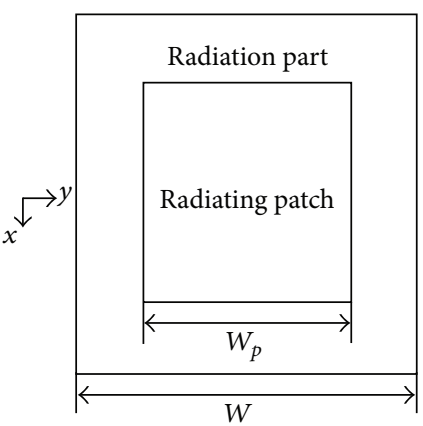

(b) Radiation part

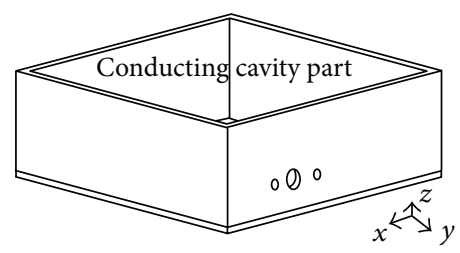

(e) Conducting cavity

FiguRE 1: Structure of the antenna.

than that of the corresponding conventional MSAs. Also, the patch size is effectively reduced to about $0.372 \lambda_{0}$, that is, only $80 \%$ of the MSA without the conducting wall. However, no experimental results and design guidelines were shown. In [9], Kim et al. presented a single-feed MSA with symmetric CP radiation and relatively large bandwidth of $4.6 \%$, but the HPBW was only about $60^{\circ}$ and the AR beamwidth was not discussed in that paper. A dual-feed circular patch antenna loaded with curved resonant strips was proposed in [11], and more than $150^{\circ} 3 \mathrm{~dB}$ power and $\mathrm{AR}$ beamwidth was achieved at the price of dramatically reduced gain and enlarged lateral sizes $\left(0.57 \lambda_{0} \times 0.57 \lambda_{0}\right)$ of the antenna.

In this paper, we present the design of a single-feed compact MSA with symmetric and wide $3 \mathrm{~dB}$ AR beamwidth and HPBW in the upper hemisphere. The configuration and design of the antenna is introduced firstly, and then parametric studies are carried out to better understand the design procedure and to optimize the geometrical sizes of the antenna. Finally, a prototype at $\mathrm{L}$ band is fabricated and tested, and good agreement is observed between the measured data and the numerical simulations.

\section{Antenna Geometry and Design}

The configuration of the proposed antenna is shown in Figure 1. It consists of four parts. (1) The radiation part (Figure $1(\mathrm{~b})$ ) is a square patch of size $W_{p}$ etched on a square dielectric substrate of permittivity $\varepsilon_{r 1}=2.25$, thickness $h_{1}$, and side length $W$. (2) The aperture control part (Figure 1(c)) is formed by four symmetrical $45^{\circ}$ isosceles trapezoids of the same size etched on the top surface of a dielectric substrate of permittivity $\varepsilon_{r 2}=2.25$, thickness $h_{2}$, and transverse size $W$. It is mounted on the top of the radiation part as a partially covered superstrate. By adjusting the top and bottom widths $W_{1}$ and $W_{2}$ of the trapezoids, the radiation aperture can be resized accordingly to control the amount of radiated energy from the patch at the radiation part. Based on our numerical simulations, this aperture control part not only can effectively increase the radiation beamwidth but also can reduce the size of the radiating patch by about $30 \%$ even if no dielectric substrate is present. (3) The feeding part (Figure $1(\mathrm{~d})$ ) consists of a series microstrip feeding line placed behind a crossedslot with a $\lambda / 4$ feeding line positioned between each arm of slot to create the phase quadrature for CP radiation. Thus, the feeding structure has the effect of multiple feed but without complicated external hybrid or power divider. The radiating patch is fed by the feeding part through an air gap of height $D_{1}$, and the coupling from the feeding part to the patch can be easily adjusted by changing $D_{1}$, the length $L_{s}$, and width $W_{s}$ of the slot. The dielectric constant, thickness, and side length of the substrate to form the feeding part are $\varepsilon_{r f}=4.4, h_{f}$, and $W$, respectively. An open tuning stub of length $L_{\text {os }}$ beyond the last arm of the slot is used to achieve impedance matching. (4) The conducting cavity part (Figure 1(e)) encompasses the edges and bottom of the former three parts to form a closed radiator as a whole. It is made of brass of thickness $T=$ $2.0 \mathrm{~mm}$ and the distance between the bottom of the cavity to the feeding layer is $D_{2}$. The inner conductor of a coaxial line is soldered to the microstrip line to feed the antenna through the side wall of the cavity, while its outer conductor 
TABLE 1: Optimized dimensions of the proposed antenna (unit: $\mathrm{mm}$ ).

\begin{tabular}{|c|c|c|c|c|c|c|c|c|c|c|c|c|c|}
\hline$W$ & $W_{1}$ & $W_{2}$ & $W_{p}$ & $T$ & $h_{1}$ & $h_{2}$ & $h_{f}$ & $D_{1}$ & $D_{2}$ & $L_{s}$ & $W_{s}$ & $L_{\text {os }}$ & $W_{f}$ \\
\hline 58.0 & 45.0 & 15.0 & 35.4 & 2.0 & 3.16 & 3.16 & 1.5 & 6.5 & 6.5 & 44.0 & 2.0 & 18.0 & 2.8 \\
\hline
\end{tabular}

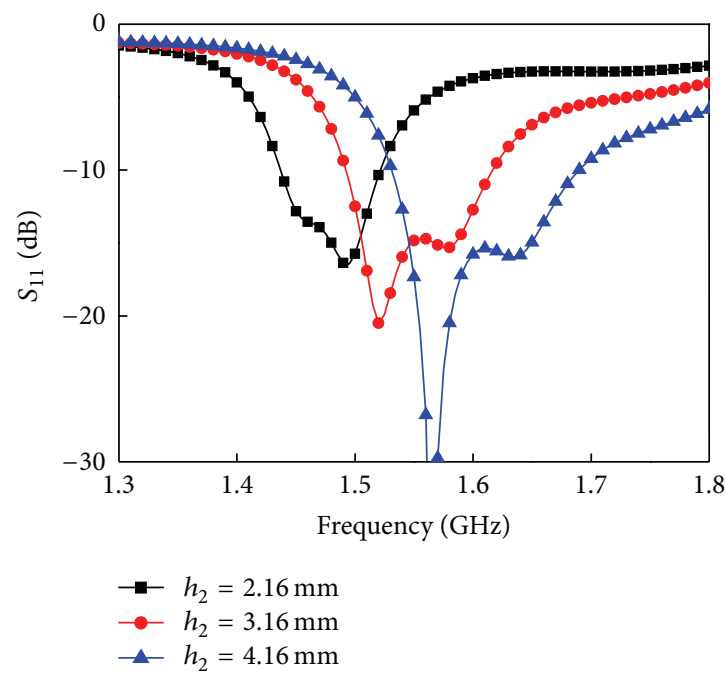

(a) Reflection coefficient $S_{11}$

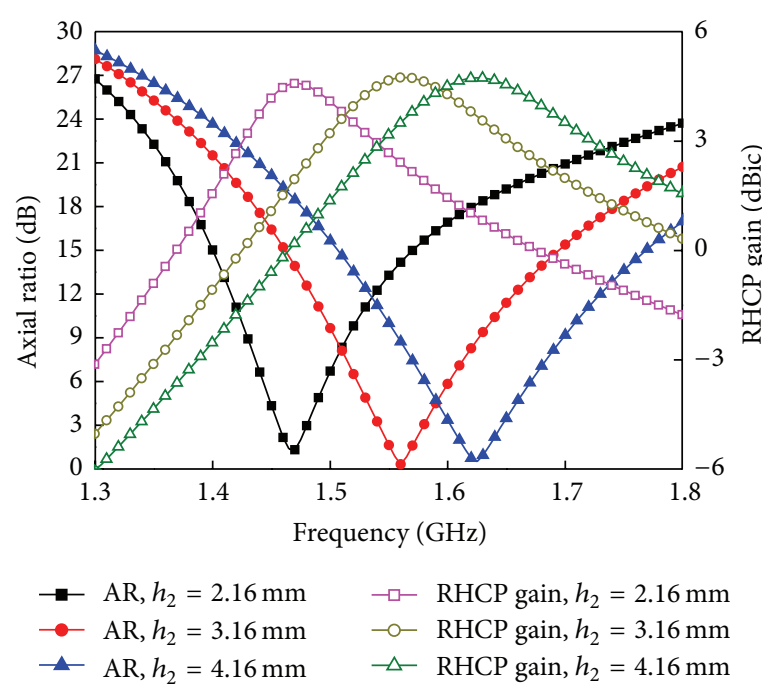

(b) AR and RHCP gain

FIGURE 2: Effect of the aperture control part thickness $h_{2}$ on the reflection coefficient, gain, and axial ratio of the antenna.

is connected to an SMA launcher that is mounted on the wall of the cavity.

As an illustration, the antenna is assumed to operate at the center frequency around $f_{c}=1.56 \mathrm{GHz}$. Due to the existence of the aperture control and conducting cavity parts, the size $W_{p}$ of the radiating patch can be reduced considerably. In [8], for a vacuum folded conducting wall, the optimum size of the patch for CP radiation is 0.372 wavelengths. Based on this criterion, we can define a size reduction factor SRF to account for the effects of the conducting cavity and aperture control part

$$
\mathrm{SRF}=\frac{0.372}{0.5} \cdot \frac{1}{\sqrt{\varepsilon_{\text {reff }}}}
$$

where $\varepsilon_{\text {reff }}$ is the effective permittivity of the dielectric filled in the cavity with the value given by $\varepsilon_{r \text { eff }}=\left(\varepsilon_{r 1}+\varepsilon_{r 1}\right) / 2$. Therefore, $W_{p}$ can be initially estimated as the product of the conventional size of patch antenna $1 / 2 \lambda_{\text {reff }}\left(\lambda_{\text {reff }}\right.$ is the wavelength in the dielectric with relative permittivity of $\varepsilon_{r \text { eff }}$ ) and SRF

$$
W_{p}=\frac{\lambda_{r \mathrm{eff}}}{2} \cdot \mathrm{SRF} \approx 32 \mathrm{~mm} .
$$

The cavity size $W$ is empirically set as double times of $W_{p}$

$$
W=2 W_{p} \approx 64 \mathrm{~mm} \text {. }
$$

With the help of ANSYS HFSS simulator, the optimized parameters of the antenna are summarized in Table 1 for further discussion.

\section{Parametric Studies}

The effects of some key geometrical sizes on the performance of the proposed CP antenna with right-hand sense are investigated in order to provide useful guidelines in the design. Note that in each parametric study, only one parameter varies and the other parameters remain unchanged in Table 1. Figure 2 shows the effect of the aperture control part thickness $h_{2}$ on the reflection coefficient, the right-hand circular polarization (RHCP) gain, and the broadside AR of the antenna. It is seen that the center operating frequency is lowered and the impedance and $3 \mathrm{~dB}$ AR bandwidths are reduced when $h_{2}$ varies from $4.16 \mathrm{~mm}$ to $2.16 \mathrm{~mm}$. The $3 \mathrm{~dB} A R$ beamwidth becomes narrow, while the values of RHCP gain and HPBW are only slightly affected by decreasing $h_{2}$ as indicated in Table 2.

As stated previously, the sizes of four trapezoids etched from edges of the top surface of the aperture control part can be used to adjust the radiation aperture of the antenna and consequently to change the radiation beamwidth. Figures 3 and 4 show the variations of antenna performance when the bottom and top lengths of the trapezoids $W_{1}$ and $W_{2}$ vary, respectively, with the base angle of the trapezoid being fixed to $45^{\circ}$. As seen, either an increased size of $W_{1}$ or a reduced size of $W_{2}$ lowers the operating frequency and decreases the RHCP gain, but at the corresponding center frequency the HPBW of the antenna has only slight variations as listed in Table 2. However, interestingly, the $3 \mathrm{~dB}$ AR beamwidth experienced relatively large changes. When the radiation aperture size becomes large by increasing $W_{2}$ or decreasing $W_{1}$, the $3 \mathrm{~dB}$ AR beamwidth is always enhanced. Therefore, 
TABLE 2: The effects of parameters $h_{2}, W_{1}, W_{2}$, and $D_{1}$ on the center operating frequency, HPBW, and $3 \mathrm{~dB}$ beamwidth of the antenna.

\begin{tabular}{lcccccccccccc}
\hline \multirow{2}{*}{ Performance } & \multicolumn{3}{c}{$h_{2}(\mathrm{~mm})$} & \multicolumn{4}{c}{$W_{1}(\mathrm{~mm})$} & \multicolumn{3}{c}{$W_{2}(\mathrm{~mm})$} & \multicolumn{3}{c}{$D_{1}(\mathrm{~mm})$} \\
& 2.16 & 3.16 & 4.16 & 40 & 45 & 50 & 10 & 15 & 20 & 5.5 & 6.5 & 7.5 \\
\hline$f_{c}(\mathrm{GHz})$ & 1.47 & 1.56 & 1.63 & 1.735 & 1.56 & 1.44 & 1.46 & 1.56 & 1.71 & 1.58 & 1.56 & 1.55 \\
RHCP gain (dBic) & 4.72 & 4.74 & 4.74 & 5.01 & 4.74 & 4.36 & 4.46 & 4.74 & 4.95 & 4.76 & 4.74 & 4.63 \\
HPBW $\left(^{\circ}\right)$ & $-55-55$ & $-55-55$ & $-55-55$ & $-53-53$ & $-55-55$ & $-56-56$ & $-56 \sim 56$ & $-55 \sim 55$ & $-54 \sim 53$ & $-55 \sim 55$ & $-55 \sim 55$ & $-54 \sim 53$ \\
$3 \mathrm{~dB} \mathrm{AR} \mathrm{coverage}\left(^{\circ}\right)$ & $-86-86$ & $-96-97$ & $-99-99$ & $-105-105$ & $-96-97$ & $-82-82$ & $-87 \sim 87$ & $-96 \sim 97$ & $-103 \sim 103$ & $-94 \sim 94$ & $-96 \sim 97$ & $-94 \sim 95$ \\
\hline
\end{tabular}

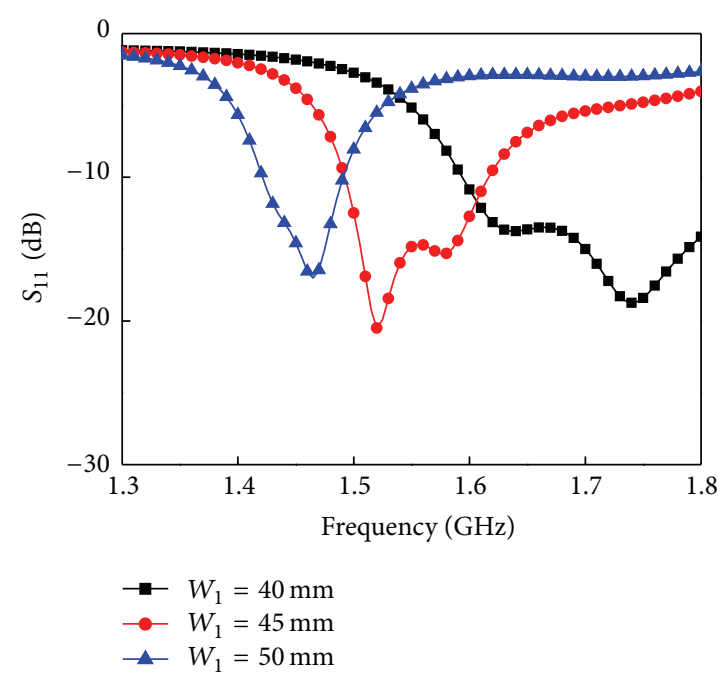

(a) Reflection coefficient $S_{11}$

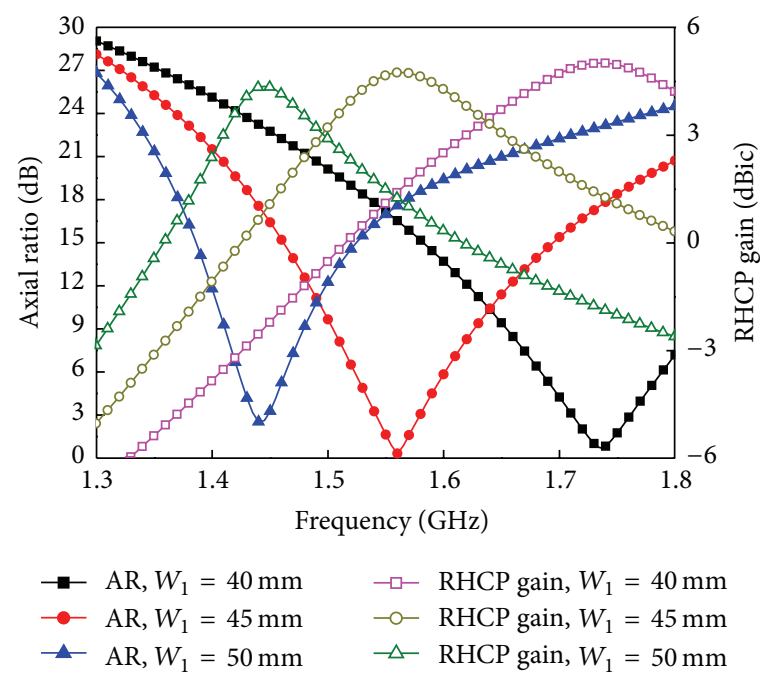

(b) AR and RHCP gain

FIGURE 3: Effect of the bottom length $W_{1}$ of the trapezoid on the reflection coefficient, gain, and axial ratio of the antenna.

we can achieve the desirable radiation properties effectively by adjusting the parameters of the aperture control part.

In Figure 5, the effect of the height of the air gap $D_{1}$ between the radiation part and feeding part on the antenna's characteristics is shown. It is seen that when $D_{1}$ is decreased the impedance bandwidth is enhanced but the CP radiation is degraded clearly. On the other hand, if the value of $D_{1}$ is too large, both the impedance and AR bandwidth will be deteriorated and the RHCP gain will be reduced due to the weak coupling between the feeding and the radiation structures. In summary, the effects of the important parameters $h_{2}, W_{2}$, $W_{1}$, and $D_{1}$ on the center operating frequency, the maximum value of RHCP gain, HPBW, and $3 \mathrm{~dB}$ AR beamwidth of the antenna are listed in Table 2.

\section{Measured Results and Discussion}

Figures 6(a) and 6(b) show the fabricated RHCP antenna with the center frequency being around $1.56 \mathrm{GHz}$. Its overall dimensions are $62 \mathrm{~mm} \times 62 \mathrm{~mm} \times 22.82 \mathrm{~mm}\left(0.32 \lambda_{0} \times\right.$ $0.32 \lambda_{0} \times 0.12 \lambda_{0}$ at $\left.1.56 \mathrm{GHz}\right)$. The antenna test system includes a vector network analyzer (VNA) HP8510C, a onedimensional rolling-plane antenna test turntable, and two L band standard horn antennas. All measurements were carried out in an anechoic chamber. The reflection coefficient measured by the VNA is shown in Figure 7. It is seen that the $10 \mathrm{~dB}$ RL bandwidth is about $6.8 \%(1.495-1.60 \mathrm{GHz})$, which is slightly narrower than the simulated results. The small discrepancy may be caused by the miss match from the SMA connector and uncertainty of the dielectric materials used in the fabrication.

The photograph of radiation pattern measurement is shown in Figure 6(c), in which the fabricated antenna was used as the transmitting antenna while the standard horn antennas were used as the receiving antennas. The horn antenna was rotated around its axis and the maximum and minimum received power $P_{\max }$ and $P_{\min }$ were recorded by the VNA, and then the value of AR in decibel can be directly obtained by $\mathrm{AR}=P_{\max }-P_{\min }$. The $\mathrm{CP}$ radiation pattern of the antenna can also be obtained by using the existing test system and the following equations:

$$
\begin{aligned}
& \left(2 E_{L}\right)^{2}=E_{1 m}^{2}+E_{2 m}^{2}-2 E_{1 m} E_{2 m} \cos \left(270^{\circ}-\Delta \varphi_{l}\right), \\
& \left(2 E_{R}\right)^{2}=E_{1 m}^{2}+E_{2 m}^{2}-2 E_{1 m} E_{2 m} \cos \left(\Delta \varphi_{l}-90^{\circ}\right),
\end{aligned}
$$

where $E_{1 m}$ and $E_{1 m}$ are magnitudes of two orthogonal linearly polarized electric field components with a phase difference $\Delta \varphi_{l}$ between them and $E_{L}$ and $E_{R}$ are magnitudes of the LHCP and RHCP components, respectively. If the received power and the phase of two linear polarization components at $\mathrm{E}$ - and $\mathrm{H}$-planes of the standard horn antenna 


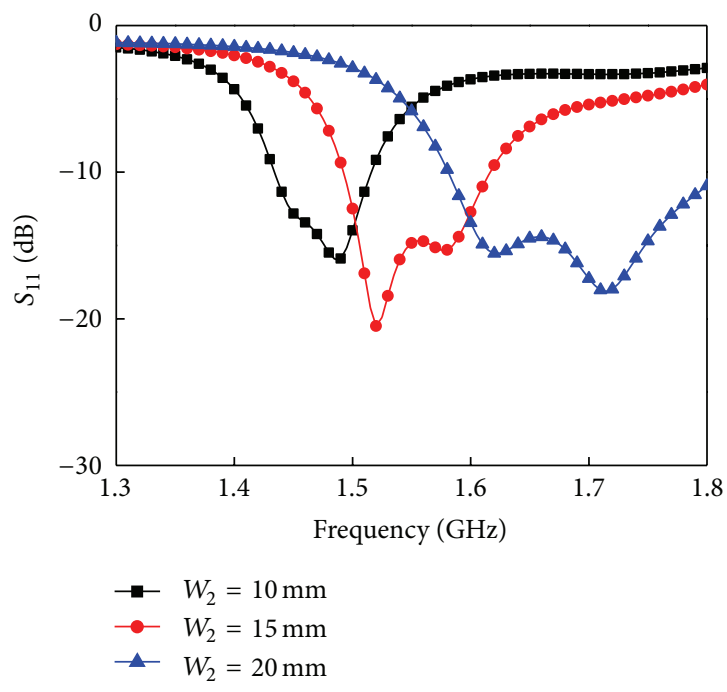

(a) Reflection coefficient $S_{11}$

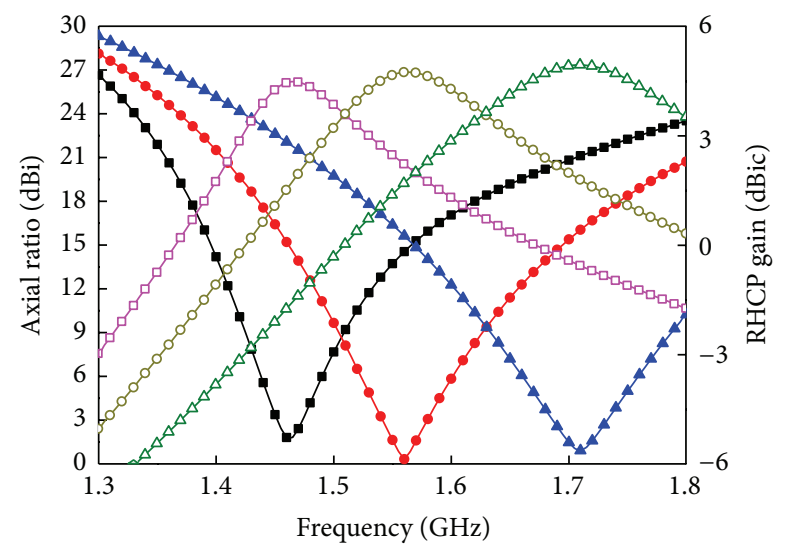

$$
\begin{array}{ll}
\rightarrow \text { AR, } W_{2}=10 \mathrm{~mm} & \square \text { RHCP gain, } W_{2}=10 \mathrm{~mm} \\
\rightarrow \text { AR, } W_{2}=15 \mathrm{~mm} & -0 \text { RHCP gain, } W_{2}=15 \mathrm{~mm} \\
-\mathrm{AR}, W_{2}=20 \mathrm{~mm} & -\triangle \mathrm{RHCP} \text { gain, } W_{2}=20 \mathrm{~mm}
\end{array}
$$

(b) AR and RHCP gain

FIGURE 4: Effect of the top length $W_{2}$ of the trapezoid on the reflection coefficient, gain, and axial ratio of the antenna.

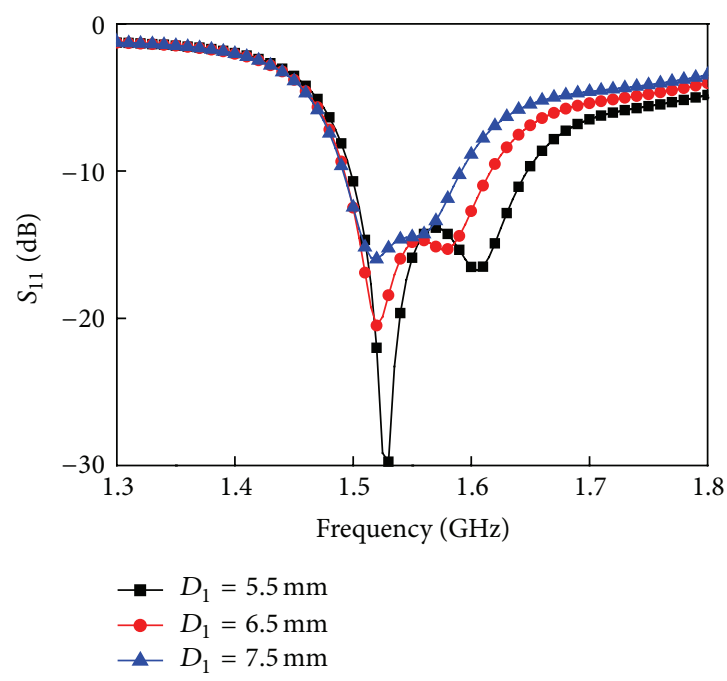

(a) Reflection coefficient $S_{11}$

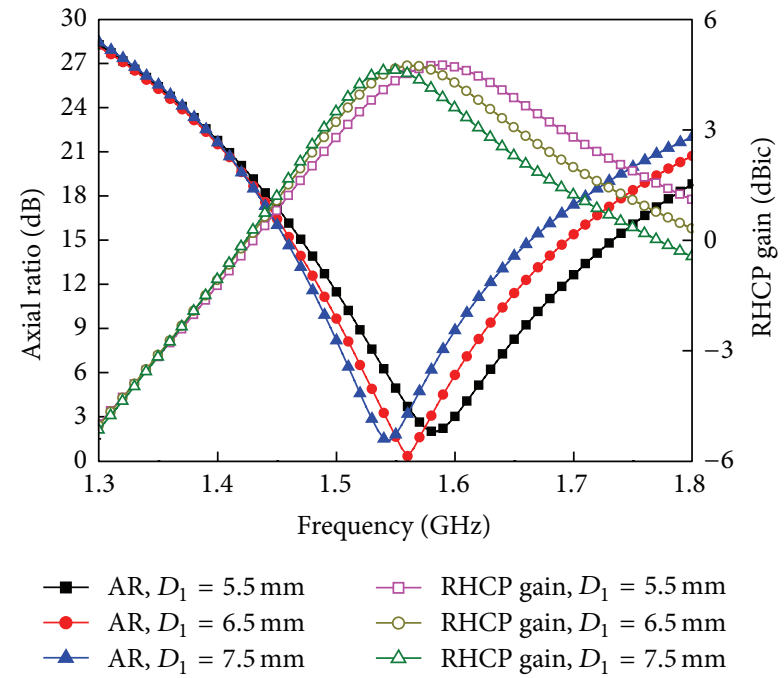

(b) AR and RHCP gain

FIGURE 5: Effect of the height of air gap $D_{1}$ on the reflection coefficient, gain, and axial ratio of the antenna.

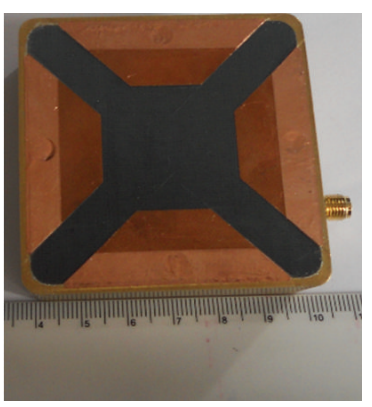

(a)

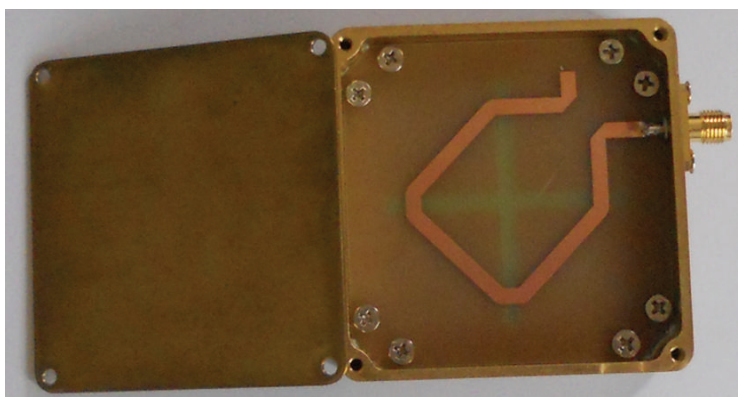

(b)

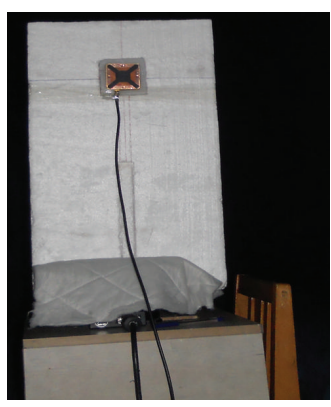

(c)

FIgURE 6: (a) top View; (b) bottom View; (c) radiation pattern measurement. 


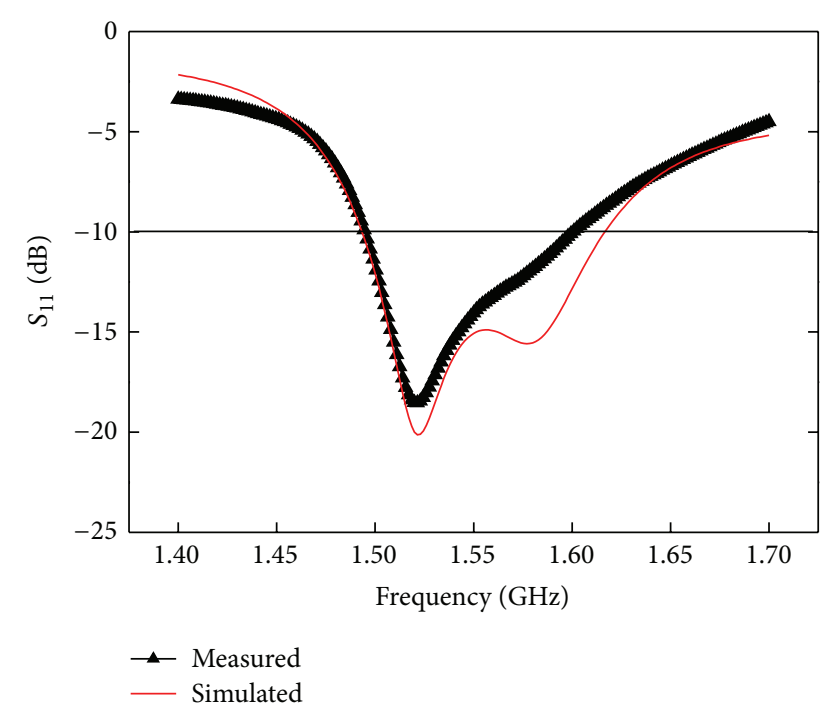

FIgURE 7: Measured and simulated reflection coefficient of the fabricated antenna.

are $P_{e}, \varphi_{e}, P_{h}$ and $\varphi_{h}$, respectively, then the LHCP and RHCP components can be expressed as

$$
\begin{gathered}
4 P_{L}=P_{e}+P_{h}-2 \sqrt{P_{e} P_{h}} \cos \left(270^{\circ}-\Delta \varphi\right), \\
4 P_{R}=P_{e}+P_{h}-2 \sqrt{P_{e} P_{h}} \cos \left(\Delta \varphi-90^{\circ}\right), \\
\Delta \varphi=\varphi_{e}-\varphi_{h} .
\end{gathered}
$$

To get RHCP gain of the antenna, two standard horn antennas must be used. First, the fabricated antenna is used as the transmitting antenna and one of the horn antennas is used as the receiving antenna, and then we obtain a set of the received power and phase of the signal $P_{e 0}, \varphi_{e 0}, P_{h 0}$, and $\varphi_{h 0}$. Next, we got another set of $P_{e 1}, \varphi_{e 1}, P_{h 1}$, and $\varphi_{h 1}$ using the other horn antenna in place of the fabricated antenna as the transmitting antenna. So we could calculate the RHCP gain by using the comparison method and (5).

Figure 8 shows the measured RHCP gain and axial ratio at broadside direction versus frequency. The measured $3 \mathrm{~dB}$ AR bandwidth is $3.0 \%(1.520-1.567 \mathrm{GHz})$, and the frequency with minimum AR is $1.544 \mathrm{GHz}$, that is, about $1 \%$ lower than the simulated one. The difference between the measured and simulated RHCP gain is less than $0.5 \mathrm{~dB}$ over the entire frequency band. It is seen that the measured gain follows the simulation, while there is a small frequency shift between the measured and simulated AR. We think that this discrepancy is due to the alignment of the transmitting and receiving antennas during the gain measurement since the measurement should be carried out two times by using the fabricated antenna as the receiving antenna first and again the standard horn antenna, while in the AR measurement only one receiving antenna is needed, which will lead to a small frequency shift between the measured gain and AR. Figures 9 and 10 depict the spatial distribution of axial ratio and the normalized radiation pattern at $1.55 \mathrm{GHz}$, respectively. The radiation pattern exhibits excellent stability with respect to

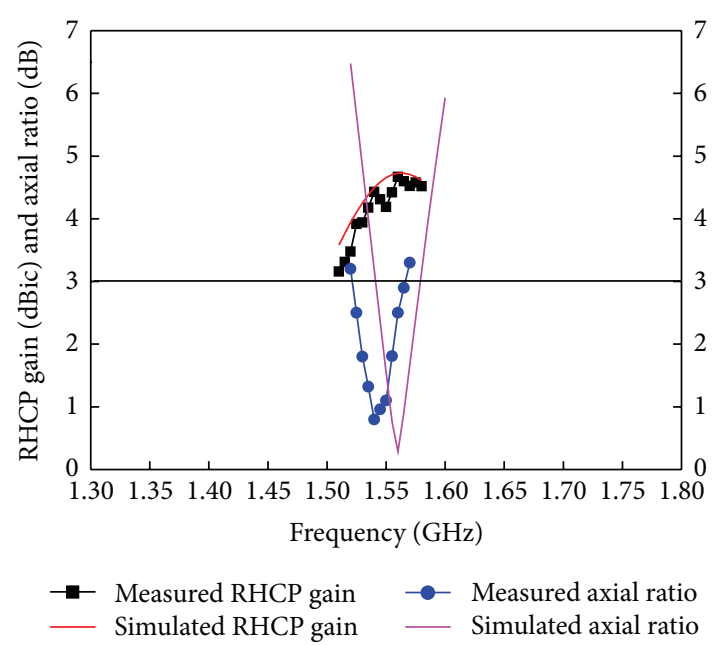

FIGURE 8: Measured and simulated RHCP gain and axial ratio of the fabricated antenna.

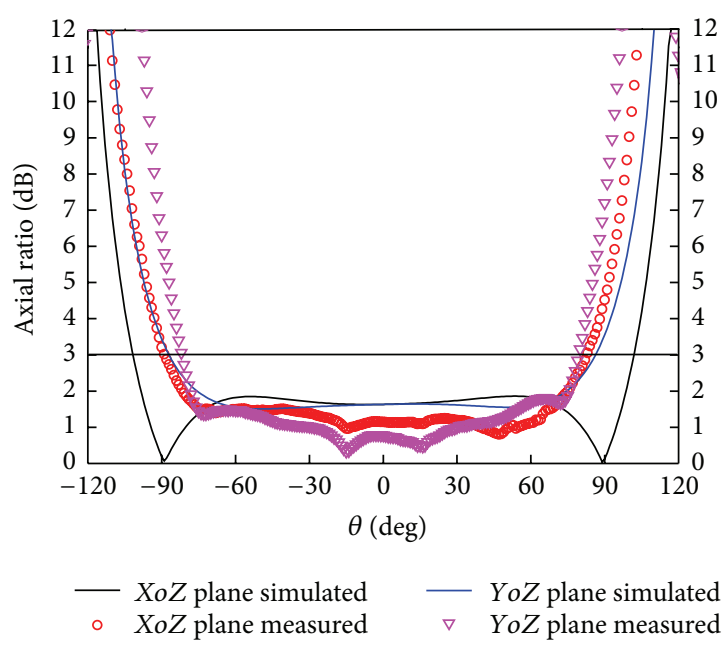

FIGURE 9: Measured and simulated axial ratio spatial distribution at $1.55 \mathrm{GHz}$.

the azimuthal angle $\varphi$, and good symmetry is also observed in the elevation planes. The average measured HPBW in $\operatorname{XoZ}\left(\varphi=0^{\circ}\right)$ and $\operatorname{YoZ}\left(\varphi=90^{\circ}\right)$ planes is $106^{\circ}$, and the $3 \mathrm{~dB}$ AR beamwidth nearly covers the entire upper half space $\left( \pm 80^{\circ}\right)$. As seen in Figures 9 and 10, the measured crosspolarization component at low elevation angle is higher than the simulated results, which may be due to the influence of the feeding coaxial line. For comparison purpose, the measured and simulated data of the return loss and axial ratio versus frequency are listed in Table 3, while the HPBW and $3 \mathrm{~dB} A R$ spatial coverage at $1.55 \mathrm{GHz}$ are shown in Table 4 .

\section{Conclusion}

A compact CP MSA with wide $3 \mathrm{~dB}$ power and axial ratio beamwidth and reasonable bandwidth is realized by the combined use of a partially covered superstrate, a conducting cavity, and a single-feed aperture coupled patch antenna 


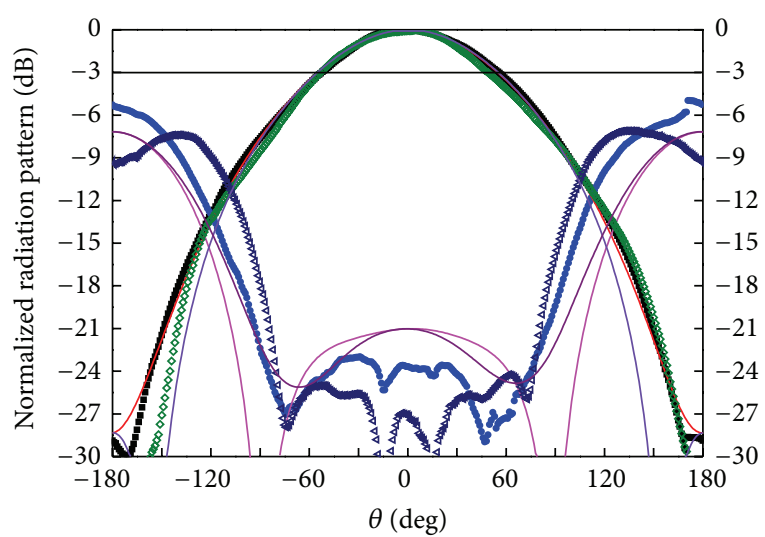

- XoZ plane measured RHCP $\diamond$ YoZ plane measured RHCP

- XoZ plane measured LHCP $\triangleleft$ YoZ plane measured LHCP

- XoZ plane simulated RHCP — Y Y

XoZ plane simulated LHCP _ $\quad$ YoZ plane simulated LHCP

FIGURE 10: Measured and simulated radiation patterns at $1.55 \mathrm{GHz}$.

TABLE 3: Measured and simulated $10 \mathrm{~dB}$ RL and $3 \mathrm{~dB}$ AR frequency range and bandwidths.

\begin{tabular}{lccc}
\hline & frequency range $(\mathrm{GHz})$ & $f_{c}(\mathrm{GHz})$ & BW (\%) \\
\hline $10 \mathrm{~dB}$ RL & & & \\
Measured & $1.495-1.600$ & 1.548 & 6.80 \\
Simulated & $1.494-1.616$ & 1.555 & 7.80 \\
$3 \mathrm{~dB}$ AR & & & \\
Measured & $1.520-1.567$ & 1.544 & 3.04 \\
Simulated & $1.541-1.579$ & 1.560 & 2.40 \\
\hline
\end{tabular}

TABLE 4: Measured and simulated HPBW and $3 \mathrm{~dB}$ AR coverage at $1.55 \mathrm{Ghz}$

\begin{tabular}{ccccc}
\hline & $\varphi=0^{\circ}$ & $\varphi=45^{\circ}$ & $\varphi=90^{\circ}$ & $\varphi=135^{\circ}$ \\
\hline HPBW $\left(^{\circ}\right)$ & & & & \\
Measured & $-53-56$ & - & $-53-50$ & - \\
Simulated & $-55-55$ & $-56-56$ & $-55-55$ & $-56-56$ \\
3 dB AR coverage $\left(^{\circ}\right)$ & & & & \\
Measured & $-89-83$ & - & $-80-79$ & - \\
Simulated & $-101-101$ & $-92-92$ & $-88-88$ & $-92-92$ \\
\hline
\end{tabular}

without using any complicated power divider. The overall dimension of the antenna is as small as $0.32 \lambda_{0} \times 0.32 \lambda_{0} \times$ $0.12 \lambda_{0}$, and the radiating patch size is only $0.19 \lambda_{0}$, that is, nearly half of the antennas in the existing literature $[8,9]$. The overlapped $10 \mathrm{~dB}$ return loss and $3 \mathrm{~dB}$ axial ratio bandwidth is $3 \%$, which is quite satisfactory when considering a single-feed MSA of such compact size. By using the aperture control part, the proposed antenna achieves very symmetrical and stable $\mathrm{CP}$ radiation almost in the entire upper hemisphere, and the average HPBW is about $106^{\circ}$ that is also much wider than that of the conventional MSA. Although only a RHCP MSA operating at $\mathrm{L}$ band is considered in this paper, the design methodology can also be used to design LHCP MSAs in other bands.

\section{Conflict of Interests}

The authors declare that there is no conflict of interests regarding the publication of this paper.

\section{References}

[1] K.-L. Wong, Compact and Broadband Microstrip Antennas, Wiley, New York, NY, USA, 2002.

[2] C.-L. Tang, J.-Y. Chiou, and K.-L. Wong, "Beamwidth enhancement of a circularly polarized microstrip antenna mounted on a three-dimensional ground structure," Microwave and Optical Technology Letters, vol. 32, no. 2, pp. 149-153, 2002.

[3] C.-W. Su, S.-K. Huang, and C.-H. Lee, "CP microstrip antenna with wide beamwidth for GPS band application," Electronics Letters, vol. 43, no. 20, pp. 1062-1063, 2007.

[4] P. C. Sharma and K. C. Gupta, "Analysis and optimized design of single feed circularly polarized microstrip antennas," IEEE Transactions on Antennas and Propagation, vol. AP-31, no. 6, pp. 949-955, 1983.

[5] K.-L. Wong and T.-W. Chiou, "Broad-band single-patch circularly polarized microstrip antenna with dual capacitively coupled feeds," IEEE Transactions on Antennas and Propagation, vol. 49, no. 1, pp. 41-44, 2001.

[6] C. Lin, F.-S. Zhang, Y.-C. Jiao, F. Zhang, and X. Xue, "A three-fed microstrip antenna for wideband circular polarization," IEEE Antennas and Wireless Propagation Letters, vol. 9, pp. 359-362, 2010.

[7] L. Bian, Y. X. Guo, L. C. Ong, and X. Q. Shi, "Wideband circularly-polarized patch antenna," IEEE Transactions on Antennas and Propagation, vol. 54, no. 9, pp. 2682-2686, 2006.

[8] H. Nakano, S. Shimada, J. Amauchi, and M. Miyata, "A circularly polarized patch antenna enclosed by a folded conducting wall," in Proceedings of the IEEE Topical Conference on Wireless Communication Technology, pp. 134-135, 2003.

[9] H. Kim, B. M. Lee, and Y. J. Yoon, "A single-feeding circularly polarized microstrip antenna with the effect of hybrid feeding," IEEE Antennas and Wireless Propagation Letters, vol. 2, pp. 7477, 2003.

[10] Z.-S. Duan, S.-B. Qu, Y. Wu, and J.-Q. Zhang, "Wide bandwidth and broad beamwidth microstrip patch antenna," Electronics Letters, vol. 45, no. 5, pp. 249-251, 2009.

[11] W. Q. Cao, B. N. Zhang, A. J. Liu et al., "A low-profile CP microstrip antenna with broad beamwidth based on loading with curved microstrip resonant structures," Journal of Electromagnetic Waves and Applications, vol. 26, no. 11-12, pp. 1602-1610, 2012. 

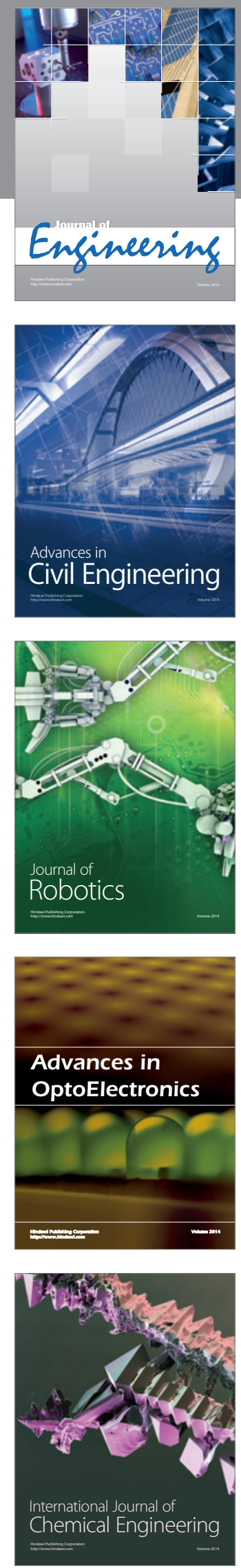

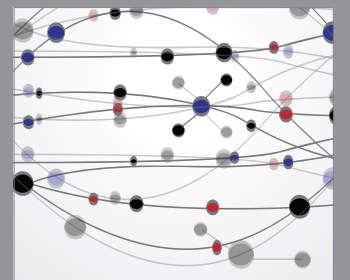

The Scientific World Journal
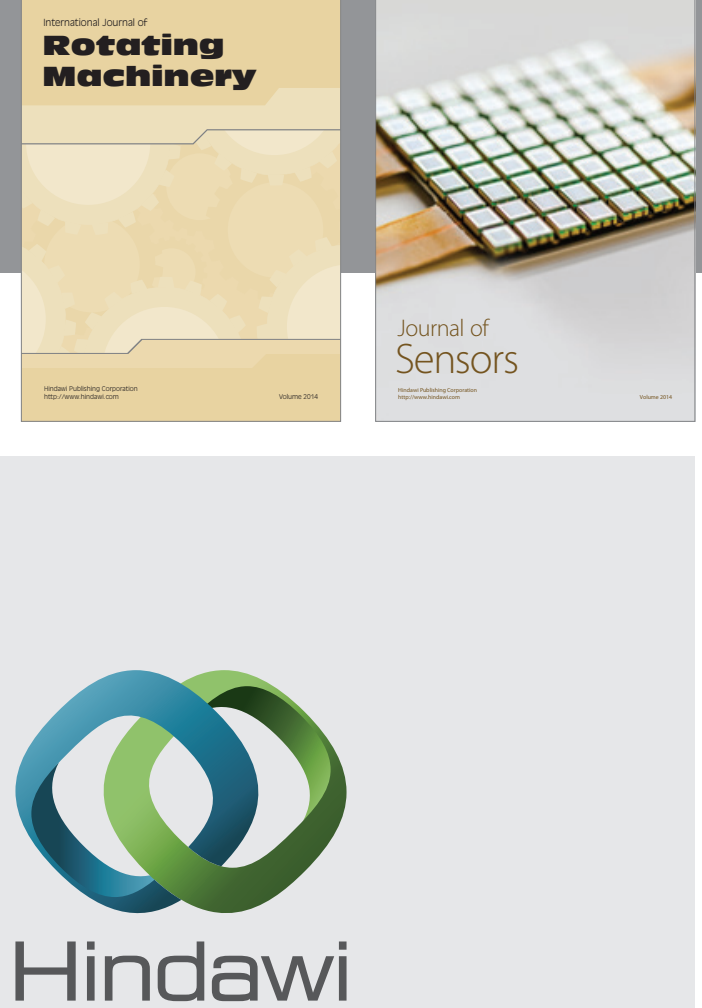

Submit your manuscripts at http://www.hindawi.com
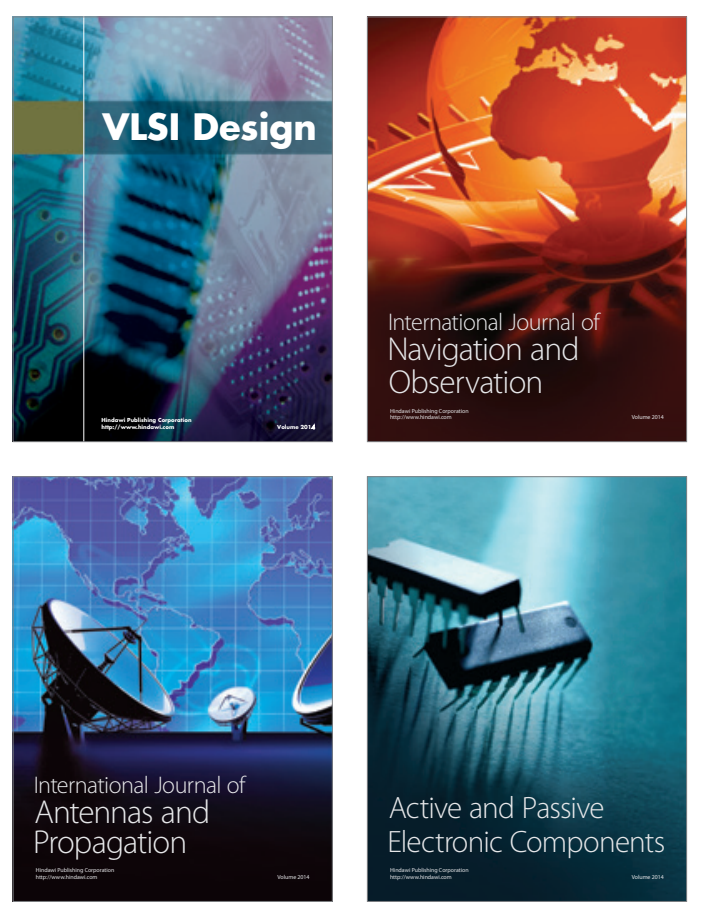
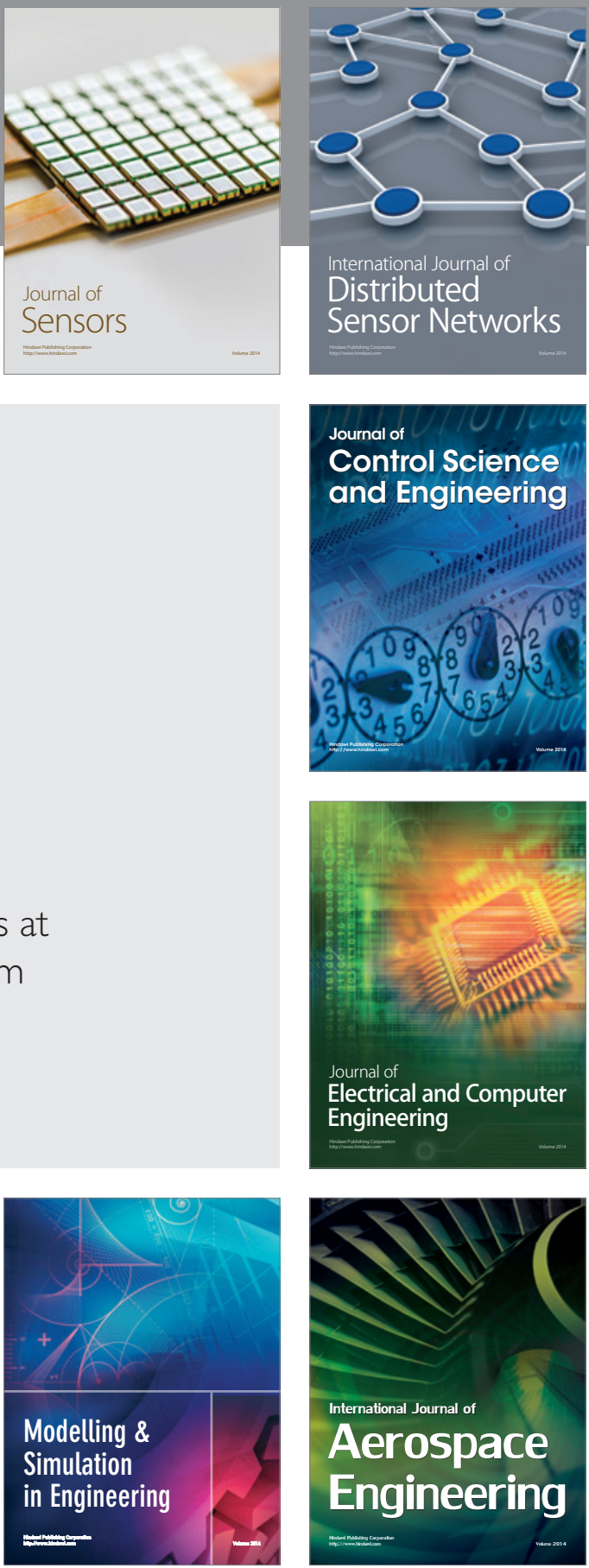

Journal of

Control Science

and Engineering
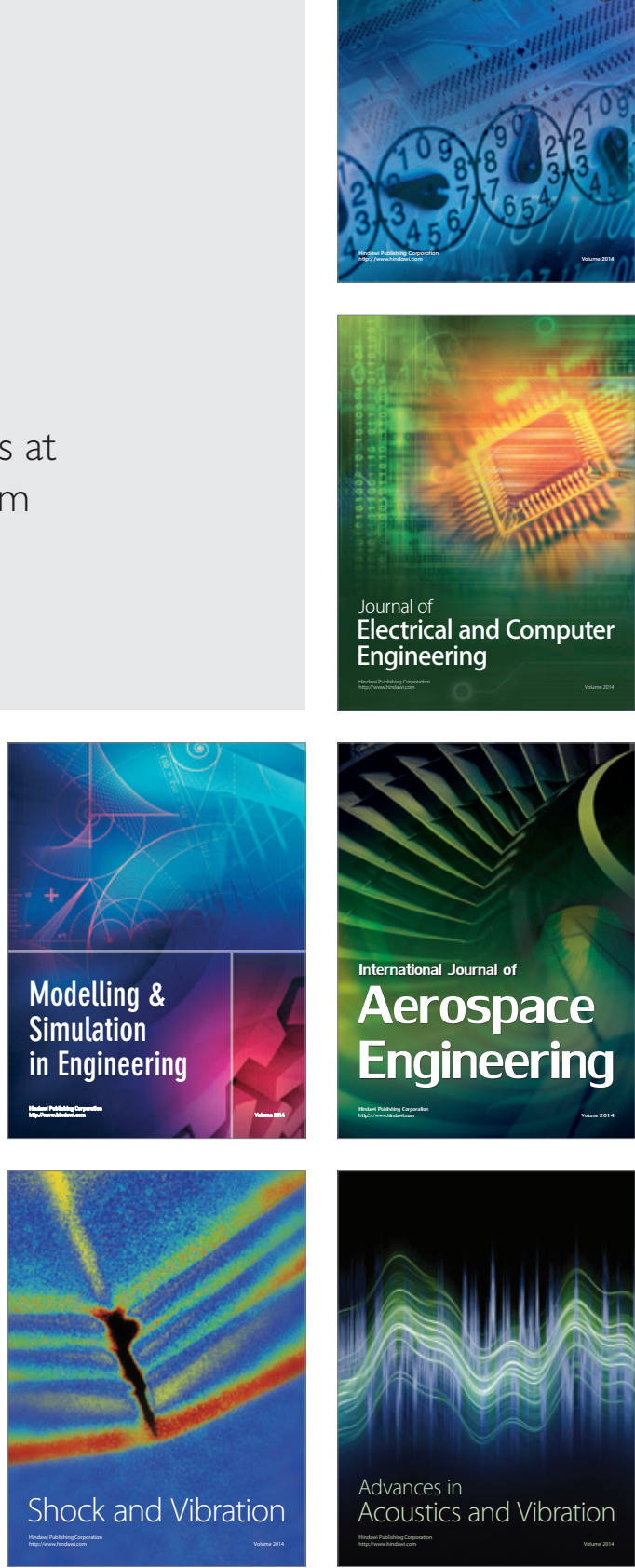